\title{
Desafios à gestão da água no contexto das alterações climáticas: a perceção de atores-chave do Baixo Vouga Lagunar
}

\author{
Challenges in water management in the context of climate \\ change: the Baixo Vouga Lagunar stakeholders' perception
}

\author{
Nuno Rodrigues', Fabiana E. P. Freitas ${ }^{2}$ Sílvia Luís ${ }^{3}$, Lisa P. Sousa ${ }^{4}$, Fátima L. Alves', Ana I. Lillebø $\varnothing^{6}$ \\ 'Bolseiro, Mestre, nuno.r.rodrigues@ua.pt; Departamento de Biologia \& Centro de Estudos do Ambiente e do Mar (CESAM), Universidade de Aveiro, Campus \\ Universitário de Santiago, 3810-193 Aveiro, Portugal \\ ${ }_{2}^{2}$ Aluna de Doutoramento, fabiana@ua.pt; Departamento de Biologia \& Centro de Estudos do Ambiente e do Mar (CESAM), Universidade de Aveiro, Campus \\ Universitário de Santiago, 3810-193 Aveiro, Portugal \\ ${ }^{3}$ Bolseira, Doutorada, silvia_luis@iscte.pt; Centro de Investigação e de Intervenção Social (CIS), ISCTE-Instituto Universitário de Lisboa, Ava das Forças Armadas, \\ 1649-026 Lisboa \\ ${ }^{4}$ Aluna de Doutoramento, lisa@ua.pt; Departamento de Ambiente e Ordenamento \& (entro de Estudos do Ambiente e do Mar (CESAM), Universidade de Aveiro, \\ Campus Universitário de Santiago, 3810-193 Aveiro, Portugal \\ ${ }_{5}^{5}$ Professora auxiliar, malves@ua.pt; Departamento de Ambiente e Ordenamento \& Centro de Estudos do Ambiente e do Mar (CESAM), Universidade de Aveiro, \\ Campus Universitário de Santiago, 3810-193 Aveiro, Portugal \\ ${ }^{6}$ Investigadora Auxiliar, Doutorada, lillebo@ua.pt; Departamento de Biologia \& Centro de Estudos do Ambiente e do Mar (CESAM), Universidade de Aveiro, Campus \\ Universitário de Santiago, 3810-193 Aveiro, Portugal
}

RESUMO: A área de estudo, o Baixo Vouga Lagunar (BVL, Portugal), situa-se na confluência da Ria de Aveiro com o rio Vouga e inclui várias linhas de água doce e de transição sob a influência da maré. Na investigação, agora apresentada, procurou-se esclarecer a problemática da gestão dos recursos hídricos no BVL, tendo em conta os possíveis efeitos das alterações climáticas $(\mathrm{AC})$ e as medidas de adaptação desejáveis. Foi feita uma análise ao conhecimento atual no que se refere às pressões (existentes e potenciais) sobre os recursos hídricos em cenários de alterações climáticas, bem como um estudo sobre a perceção dos atores-chave do BVL sobre os impactes das AC, através de 18 entrevistas. A análise das entrevistas permitiu verificar que as AC não constituem a maior preocupação no BVL e que o impacte causado é bem menor do que o atribuído a outros fatores, nomeadamente alterações na geomorfologia da laguna. $O$ estudo mostrou que os problemas existentes no BVL (intrusão salina, inundações, entre outros) apresentam-se como prioritários e daí a necessidade da sua consideração na formulação de políticas públicas, independentemente de decorrerem diretamente ou indiretamente das AC.

Palavras-chave: recursos hídricos; perceção de risco; envolvimento; Ria de Aveiro; subida do nível do mar; ecossistemas estuarinos.

ABSTRACT: The study area, Baixo Vouga Lagunar (BVL, Portugal), is located in Ria de Aveiro confluence with Vouga river and it includes several fresh and transition watercourses under tidal influence. This research aimed for unravel the water management issue in BVL, considering the possible climate change (CC) effects and the desirable adaptation measures. An analysis was conducted considering the knowledge on the current and the potential pressures over the water resources, in the context of CC. A study on stakeholders' perception over the CC impacts was also performed, through 18 interviews. The analysis of the interviews acknowledges that CC are not the main concern in BVL and that the impacts were considerable lower than the one attributed to other factors, namely by the changes in the lagoon geomorphology. The study showed that the current BVL issues (saline intrusion, flooding, among others) were considered priority and then the necessity for including them in public policies development, regardless if they are directly or indirectly due to the CC.

Keywords: water resources; risk perception; involvement; Ria de Aveiro; sea level rise; estuarine ecosystems.

O texto deste artigo foi submetido para revisão e possível publicação em fevereiro de 2016, tendo sido aceite pela Comissão de Editores Científicos Associados em abril de 2016. Este artigo é parte integrante da Revista Recursos Hídricos, Vol. 37, № 2, 49-63, outubro de 2016.

(c) APRH, ISSN 0870-1741 | DOI 10.5894/rh37n2-cti2

Recursos Hídricos $\mid 49$ 


\section{INTRODUÇÃO}

A gestão integrada da água em Portugal apresenta-se como um desafio na medida em que é necessário ter em consideração a variabilidade espacial dos recursos hídricos e as infraestruturas associadas, assim como gerir interesses e conflitos, estabelecer prioridades, definir políticas e estabelecer instrumentos de planeamento (Fidélis e Roebeling, 2014; Sousa et al., 2015). Adicionalmente, é expectável que as consequências das alterações climáticas (AC) afetem os recursos hídricos ao nível das bacias hidrográficas que confluem para os ecossistemas costeiros, devido a alterações na precipitação, evapotranspiração potencial e temperatura (Stefanova et al., 2014).

\subsection{A Gestão da Água em Portugal}

A Lei da Água (Lei n58/2005, de 29 de dezembro alterada pelo Decreto-Lei no 245/2009, de 22 de setembro e pelo Decreto-Lei no 130/2012, de 22 de junho) estabelece as bases para a gestão dos recursos hídricos superficiais, de transição, costeiros e subterrâneos em Portugal. A autoridade nacional com responsabilidades na gestão da água é a Agência Portuguesa do Ambiente (APA, I.P.). Compete à APA desenvolver e implementar o Plano Nacional da Água, os Planos de Gestão de Bacia Hidrográfica e os Planos Específicos de Gestão de Águas. A utilização da água por privados, nos casos em que potencialmente haja um impacte relevante sobre este recurso, requer uma apreciação por parte da APA e a posterior emissão de um título de utilização. Cabe às Comissões de Coordenação e Desenvolvimento Regional a proteção e valorização dos recursos hídricos através do exercício dos instrumentos de gestão territorial (Decreto-Lei 130/2012, de 22 de junho).

\subsection{Alterações climáticas em zonas estuarinas}

Globalmente, as zonas costeiras e em particular as zonas estuarinas encontram-se sujeitas a pressões decorrentes da atividade humana, mas também a eventos naturais extremos, nomeadamente de origem marítima e fluvial, como as tempestades, o vento, as marés e a subida do nível da maré (IPCC, 2014a; EEA, 2015).
Os efeitos das AC têm também contribuído para o agravamento das pressões naturais sobre os ecossistemas costeiros, nomeadamente pela subida do nível médio do mar, pelas alterações da temperatura e pela acidificação dos oceanos (IPCC, 2014a).

Neste contexto, é expectável que alterações nos parâmetros climáticos influenciem os riscos associados ao clima, com impacte para a sociedade e para o ambiente. Para a região mediterrânea, onde se inclui Portugal, os impactes esperados identificados são: a seca; o aumento das ondas de calor (em duração e intensidade); a escassez de água doce; e a subida do nível médio do mar (IPCC, 2014a; EEA, 2015).

Outros impactes esperados para Portugal incluem: o aumento da precipitação durante o inverno, contrapondoa uma diminuiçãodurante o verão; a erosão costeira e o consequente recuo da linha de costa; o aumento do risco de inundação costeira; e a ampliação da intrusão salina nos estuários e zonas adjacentes (Santos e Miranda, 2006; RCM n²4/2010, de 1 de Abril; Schimdt et al., 2012). Os impactes decorrentes da subida do nível médio do mar incluem a inundação costeira das zonas de topografia baixa, a intrusão salina superficial nos estuários, a erosão costeira, os galgamentos oceânicos e a perda de habitats (IPCC, 2014a; Santos et al., 2014; EEA, 2015).

As políticas de adaptação deverão ser flexíveis, de forma a lidar com a incerteza associada às potenciais consequências das alterações climáticas e ao horizonte de longo prazo, assegurando assim benefícios perante diferentes cenários. A incerteza não deverá ser pretexto para uma política de não antecipar a adaptação aos impactes que irão surgir (IPCC, 2014a; EEA, 2015). .

\subsection{Adaptação às alterações climáticas em Portugal}

Em linha com a política europeia de $A C$, em 2010, Portugal aprovou a Estratégia Nacional de Adaptação às Alterações Climáticas (ENAAC; RCM No24/2010, de 1 de abril), que sintetiza as principais alterações referentes ao clima durante o século XX, sumariando os cenários e as projeções climáticas para Portugal. A Tabela 1 resume a estrutura definida na ENAAC. Para cada 
setor foram constituídos grupos de trabalho por forma a efetuar uma análise mais focada sobre cada tema em particular. No que se refere aos setores recursos hídricos e zonas costeiras as medidas propostas incluem: a melhoria dos sistemas de monitorização, previsão e alerta (e.g. instrumentos de gestão do risco como o Sistema Nacional de Informação sobre os Recursos Hídricos, o Sistema de Licenciamento de Recursos Hídricos e modelos numéricos que permitam antever situações críticas); a identificação das zonas e a avaliação dos fatores de risco referentes a cheias e inundações; o reforço das infraestruturas de proteção de cheias; o esclarecimento na tomada de decisão de medidas de recuo e abandono versus proteção; e o estudo da vulnerabilidade dos aquíferos costeiros à intrusão salina (Canaveira e Papudo, 2013).

\subsection{Baixo Vouga Lagunar}

O presente estudo pretende discutir a problemática da gestão dos recursos hídricos no contexto das alterações climáticas, utilizando como caso de estudo o Baixo Vouga Lagunar (BVL - Figura 1) e considerando as pressões a que está sujeito.

A metodologia de trabalho consistiu: na análise de estudos e projetos existentes; na identificação das pressões atuais e dos impactos potenciais das $A C$ sobre o $B V L$, particularmente sobre os recursos hídricos; na análise das políticas, estratégias e planos; e na análise de entrevistas semiestruturadas conduzidas no âmbito do projeto ADAPT-MED a diferentes atores-chave.

\section{MÉTODOS}

\subsection{Caso de estudo}

O mosaico territorial do BVL resulta da estreita relação entre o Homem, o Território e a Água. A presença do Homem, através da agricultura, moldou a paisagem de acordo com os objetivos de produção e de sustentabilidade económica (Andresen e Curado, 2001). No entanto, as

Tabela 1. Estrutura da ENAAC (fonte: RCM No24/2010).

\begin{tabular}{|c|c|c|}
\hline Instrumento & Âmbito & Responsabilidade de aplicação \\
\hline Estratégia Nacional de Adaptação às Alterações Climáticas & Estratégico & APA e entidades setoriais \\
\hline \multicolumn{3}{|l|}{ Objetivos } \\
\hline \multicolumn{3}{|c|}{$\begin{array}{l}\text { - Informação e conhecimento } \\
\text { - } \quad \text { Redução da vulnerabilidade e aumento da capacidade de resposta } \\
\text { - } \\
\text { - } \quad \text { Coopticipação, sensibilização e divulgação } \\
\text { - internacional. }\end{array}$} \\
\hline \multicolumn{3}{|c|}{ Metodologia para a identificação e aplicação de ações de adaptação } \\
\hline \multicolumn{3}{|c|}{$\begin{array}{l}\text { - } \quad \text { Definição de cenários climáticos e socioecómicos } \\
\text { - } \quad \text { Identificação de riscos e impactes } \\
\text { - } \quad \text { Definição de medidas de adaptação } \\
\text { - Implementação de medidas } \\
\text { - Elaboração e revisão estratégica }\end{array}$} \\
\hline \multicolumn{3}{|l|}{ Setores estratégicos } \\
\hline $\begin{array}{l}\text { - } \text { Ordenamento do território e cidades } \\
\text { - } \quad \text { Recursos hídricos } \\
\text { - } \quad \text { Segurança de pessoas e bens } \\
\text { - } \quad \text { Saúde humana } \\
\text { - } \quad \text { Energia e indústria } \\
\text { - } \quad \text { Turismo } \\
\text { - } \quad \text { Agricultura, florestas e pescas } \\
\text { - Zonas costeiras } \\
\text { - } \quad \text { Biodiversidade }\end{array}$ & & \\
\hline
\end{tabular}




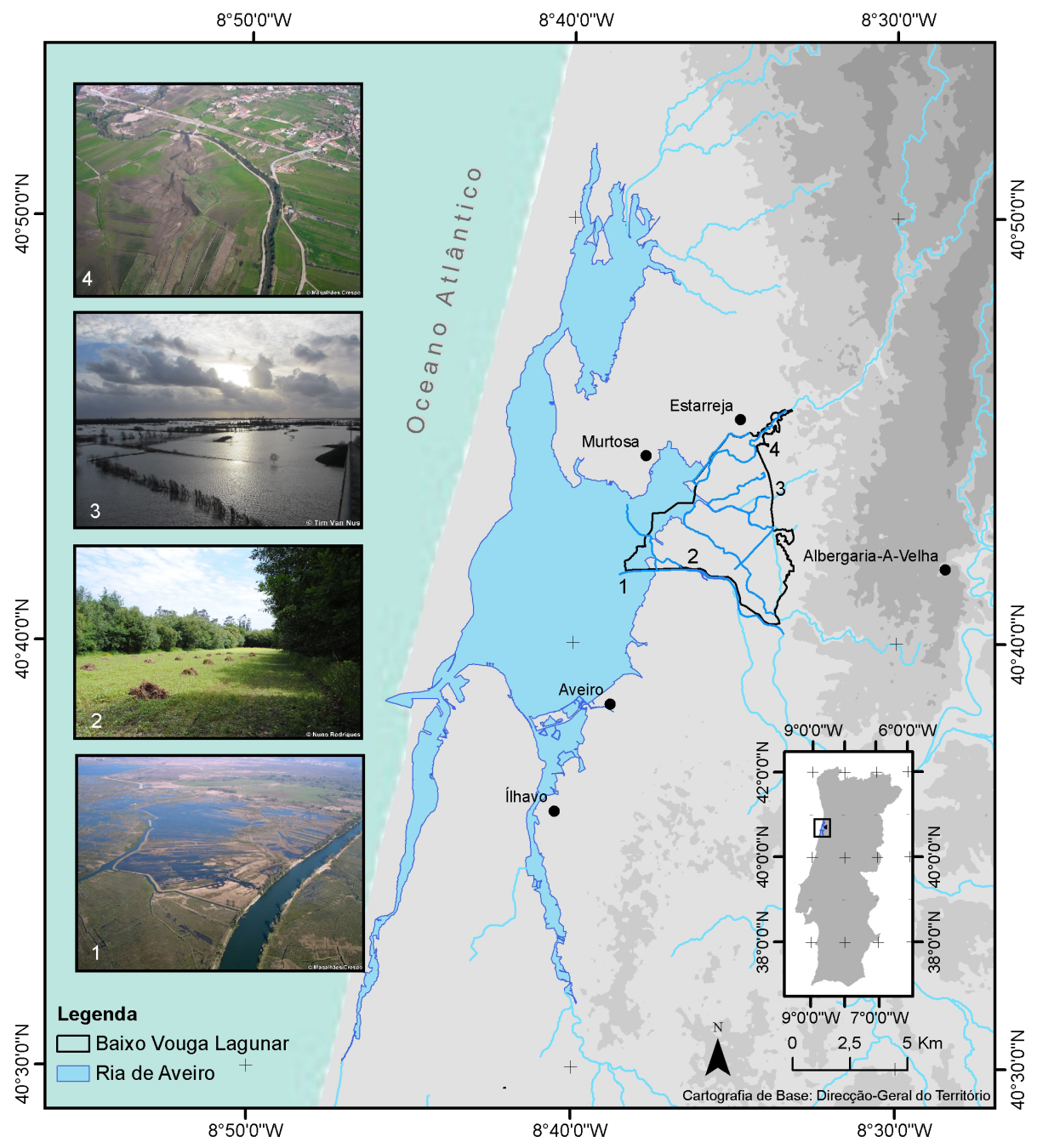

Figura 1. Localização do Baixo Vouga Lagunar e da Ria de Aveiro (fonte: APA, I.P /ARH Centro).

frequentes inundações e o aumento da intrusão salina superficial (proveniente da laguna) ameaçam constantemente este sistema. A zona de confluência entre o rio Vouga e a Ria de Aveiro situa-se na parte sudoeste do BVL, cujo sistema hídrico inclui linhas de água de baixa profundidade sob a influência da maré (águas de transição) e linhas dulçaquícolas (ver Figura 2) (ADAPT-MED, 2015a). O regime hídrico no BVL é significativamente diferenciado, sendo que recebe menos de 2 milhões $\mathrm{m}^{3}$ de água doce ( $2 / 3$ provenientes do rio Vouga), enquanto que de jusante recebe cerca de 25 a 90 milhões $\mathrm{m}^{3}$ de água de proveniente da laguna (da Ria de Aveiro) (LAGOONS, 2013).

Os ecossistemas presentes no BVL (em zonas húmidas, floresta, campo aberto e 'Bocage'1) fornecem um conjunto de bens e serviços essenciais para o bem-estar humano e para as atividades económicas. A presença da água no BVL, tanto doce como de transição, é de extrema importância para a manutenção de ecossistemas aquáticos, em pauis e sapais sobre

1 - O 'Bocage' é um ecossistema único nesta região que consiste em parcelas de pequena dimensão, compartimentadas por sebes vivas de folhosas e/ ou arbustos (e.g. salgueiro - Salix sp., amieiro - Alnus glutinosa, carvalho-vermelho - Quercus robur) e por valas de distribuição/escoamento de água. Esta paisagem moldada pelo Homem traduz-se num sistema de irrigação muito particular gerido pelos agricultores, utilizado na manutenção das pastagens e na produção de milho (Zea mays) e forragens (ADAPT-MED, 2015a). 
a influência da maré, e para a manutenção das atividades agrícolas que aqui ocorrem.

\subsection{Os possíveis impactes sobre 0 BVL em consequência das alterações climáticas}

Com base na literatura existente, sobre os possíveis efeitos das AC na costa portuguesa e na Ria de Aveiro, foi possível percecionar se o BVL será particularmente afetado em consequência da subida do nível do mar e de inundações (fluviais e marítimas), devido a eventos extremos (Lopes, et al., 2011; Lopes, Azevedo, \& Dias, Flooding assessment under sea level rise scenarios: Ria de Aveiro case study, 2013; LAGOONS, 2014).

\subsection{Entrevistas semiestruturadas}

Por forma a avaliar a perceção dos atores-chave do $B V L$, no que concerne às $A C$ a nível local e assim promover o seu envolvimento nesta temática, foram conduzidas dezoito entrevistas presenciais, entre junho e julho de 2014, com instituições da administração central e regional (e.g. APA I.P., CCDR-C, DRAP-C), municípios, juntas de freguesia, associações de utilizadores (e.g. agricultura, caça e pesca), entidades não-governamentais e empresas privadas (ADAPT-MED, 2015b). O guião de entrevista era semiestruturado, incluiu um conjunto predefinido de questões de resposta aberta, que permitiu abordar os diferentes temas e obter a opinião dos entrevistados. Durante as entrevistas os atores-chave foram questionados sobre: a relação (pessoal e institucional) com as alterações climáticas e com as políticas de adaptação, procurando-se saber de que forma poderiam contribuir para o processo de adaptação; as alterações climáticas em geral e no

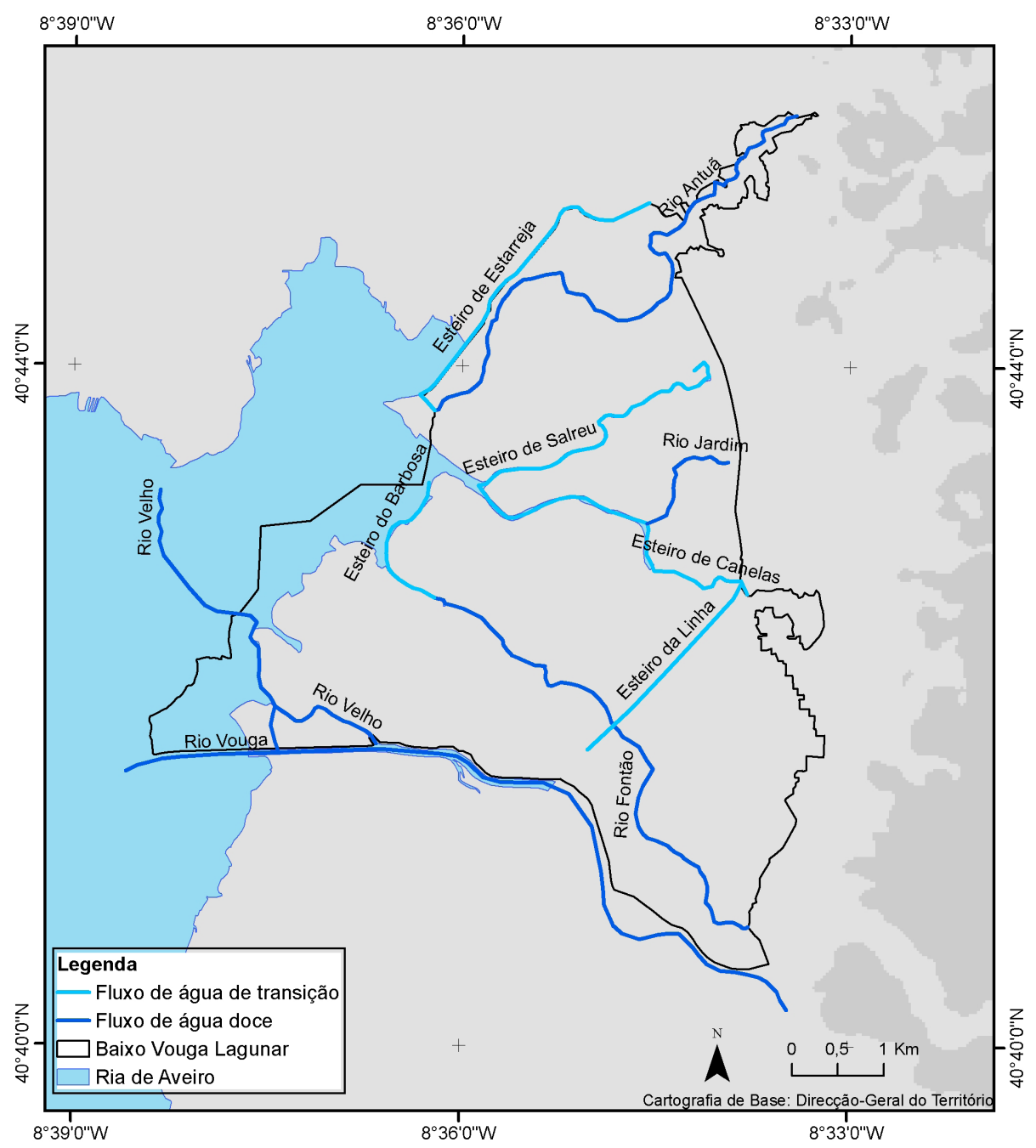

Figura 2. Principais linhas de água doce e de transição no BVL

(fonte: APA, I.P /ARH Centro). 
caso concreto do BVL, descriminando quais os impactes negativos e positivos deste fenómeno e sobre quais dariam maior prioridade; quais as políticas que atualmente integram medidas de adaptação; as suas perceções de como tem sido conduzido processo de desenvolvimento de políticas, percebendo se estiveram envolvidos e se identificavam os insucessos e sucessos das políticas; quais as evoluções esperadas, identificando boas políticas de adaptação no horizonte 2050 e, nas suas opiniões, de que forma as alterações climáticas poderiam ser integradas na formulação de políticas; e se necessitavam de mais informação por forma a discutirem e estarem envolvidos no processo adaptação às AC.

\section{RESULTADOS E DISCUSSÃO}

\subsection{Evidências das alterações climáticas e de eventos extremos sobre o Baixo Vouga Lagunar}

A região de Aveiro, integrada na zona costeira, encontra-se exposta aos impactes da subida do nível médio do mar. Estudos recentes registaram para esta região uma subida do nível médio do mar de 1.15 +/- $0.68 \mathrm{~mm}$ por ano, durante o período de 1976 a 2003 (Lopes et al., 2011). Dado que o BVL se encontra ligado ao oceano Atlântico, através da Ria de Aveiro, existe um fluxo permanente de água salgada nos esteiros, devido à ação da maré, como é ilustrado na Figura 2. Assim, é expectável que o BVL possa ser afetado pelas alterações no nível médio do mar, através da Ria de Aveiro (Lopes et al., 2013). De montante, as pressões ou ameaças estarão associadas, essencialmente, a alterações no ciclo hidrológico do rio Vouga, devido a variações sazonais e anuais da precipitação (Stefanova et al., 2014).

No caso particular da Ria de Aveiro, Picado et al. (2010) reconhecem que outros fatores, que não as $A C$, serão também responsáveis pelas alterações na dinâmica das marés, nomeadamente a falta de conservação e manutenção de infraestruturas costeiras e as alterações na geomorfologia da laguna. De facto, durante as últimas décadas houve várias intervenções na bacia hidrográfica do Vouga, nomeadamente na Ria de Aveiro, que influenciaram o equilíbrio entre a água doce e de transição na zona mais a jusante do BVL. De destacar, as obras de dragagem do Porto de Aveiro $^{2}$ e nos canais por forma a melhorar a navegabilidade, e a colocação de quebra-mares ao longo da costa (Silva et al., 2004). Os efeitos observados incluem alterações na velocidade da água e nas correntes, alterações nos padrões espaciais em termos de erosão e acumulação de sedimentos, e no aumento do prisma da maré (Picado et al., 2010). Já Andresen, Curado (2001) reconheciam que as obras de dragagem da barra contribuíram para as alterações no prisma da maré e identificavam que essas mesmas alterações já estavam previstas no estudo de impacte ambiental do "Projecto de Desassoreamento da Ria de Aveiro 1a Fase", elaborado em 1993.

As alterações no prisma da maré e no aumento da velocidade da água potenciaram a duração dos eventos de intrusão salina, nomeadamente durante as marés-vivas, colocando os ecossistemas do BVL sobre maior pressão (LAGOONS, 2013). Entre 1995 e 1999, um dique de proteção de marés (de betão) com $4 \mathrm{~km}$ foi parcialmente construído na frente oeste do BVL por forma a reduzir o risco de intrusão salina e de inundações de origem costeira.

$\mathrm{Na}$ região da Ria de Aveiro ocorrem, frequentemente, inundações fluviais de montante devido a fenómenos climáticos extremos (Lopes et al., 2013). Especificamente no BVL, em situações de precipitação excessiva, o caudal dos rios Vouga e Antuã aumenta ao ponto de a água galgar as margens e inundar os campos agrícolas (Figura 3). Nas situações em que se conjugam simultaneamente a precipitação intensa e os eventos marítimos extremos (e.g. marés vivas, tempestades), as inundações (de água doce ou de transição) afetam uma maior extensão (Alves et al., 2013a; ADAPT-MED, 2015a). Em consequência das inundações ocorre frequentemente 0 colapso das margens levando à salinização dos terrenos agrícolas e à morte das sebes arbóreas da paisagem de 'Bocage'- Figura 4 e

2 - Entre 1988 e 1999 o canal de entrada na barra de Aveiro foi dragado por forma a assegurar a batimétrica de $10 \mathrm{~m}$. Desde então só existiram obras de dragagem por forma a manter esta profundidade (Jorge Rua, Comunicação Pessoal, 2014). 
Figura 5. Outras consequências das inundações extremas incluem o colapso de infraestruturas como diques secundários, estradas, caminhos e pontes.

A gestão diária da água no BVL é maioritariamente realizada pelos agricultores, que são responsáveis pela abertura e fecho de comportas manuais, na rede de valas e canais (apoiados também por comportas de maré) (ADAPT-MED, 2015a). Durante o verão são instalados diques temporários no rio Vouga com o objetivo de fazer subir o nível freático e, desta forma, permitir que a água flua naturalmente para a rede de valas. Estes diques têm igualmente como função diminuir o efeito da cunha salina e garantir o fornecimento de água para a indústria aí presente (ARH Centro, 2012).

\subsection{Cenários futuros para 0 BVL em consequência das alterações climáticas}

Baseado no cenário de emissões $A 2^{3}$, elaborado

3 - A família de cenários A2 do IPCC descrevem um mundo muito heterogéneo, em que a população global aumenta continuamente e o crescimento económico regional é mais fragmentado e mais lento que as outras famílias de cenários (IPCC, 2014b). pelo IPCC, Lopes et al. (2011) estimaram para o final do século, para a zona costeira portuguesa, um aumento do nível médio do mar de 0,42m que, devido à incerteza associada, poderá chegar a 0,64m. Isto significa que, de acordo com Lopes et al. (2011), a área inundável da Ria de Aveiro poderá aumentar dos atuais $88,6 \mathrm{~km} 2$ para $107,5 \mathrm{~km} 2$ e para $118,6 \mathrm{~km} 2$. Isto é, um aumento de $23 \%$ e $35 \%$ respetivamente, sendo a diferença média atual da área alagada entre maré vazia e a maré cheia é de $20 \%$. É de salvaguardar que estes cenários de inundação, resultantes da subida do nível médio do mar, não têm em conta os processos biogeomorfológicos, como a taxa de sedimentação e a taxa de acreção dos ecossistemas de sapal, que poderão impedir a progressão das águas marinhas. No entanto, estas previsões evidenciam a suscetibilidade das zonas de baixa elevação, como o BVL, à intrusão salina superficial, comprometendo não só as atuais infraestruturas e atividades, como as áreas residenciais e os campos agrícolas (Lopes et al., 2013). A Figura 6 ilustra as simulações de inundação na Ria de Aveiro, com detalhe para a região do $\mathrm{BVL}$, para o cenário de ano típico de em três períodos distintos (1981-2010, 2011-2040, 2071-2098),

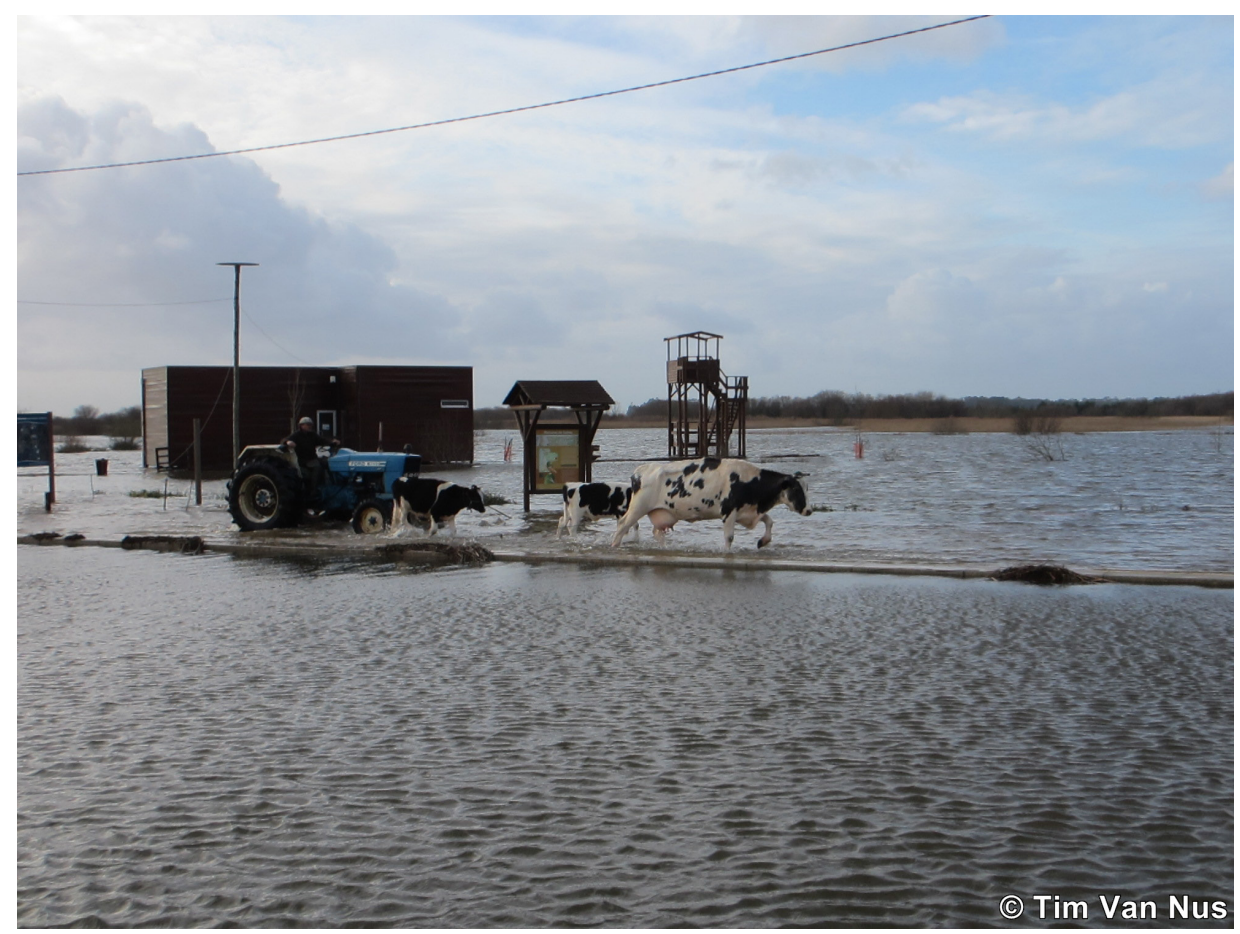

Figura 3. Inundações junto ao Esteiro de Salreu por galgamento das linhas de água, em janeiro de 2014. 


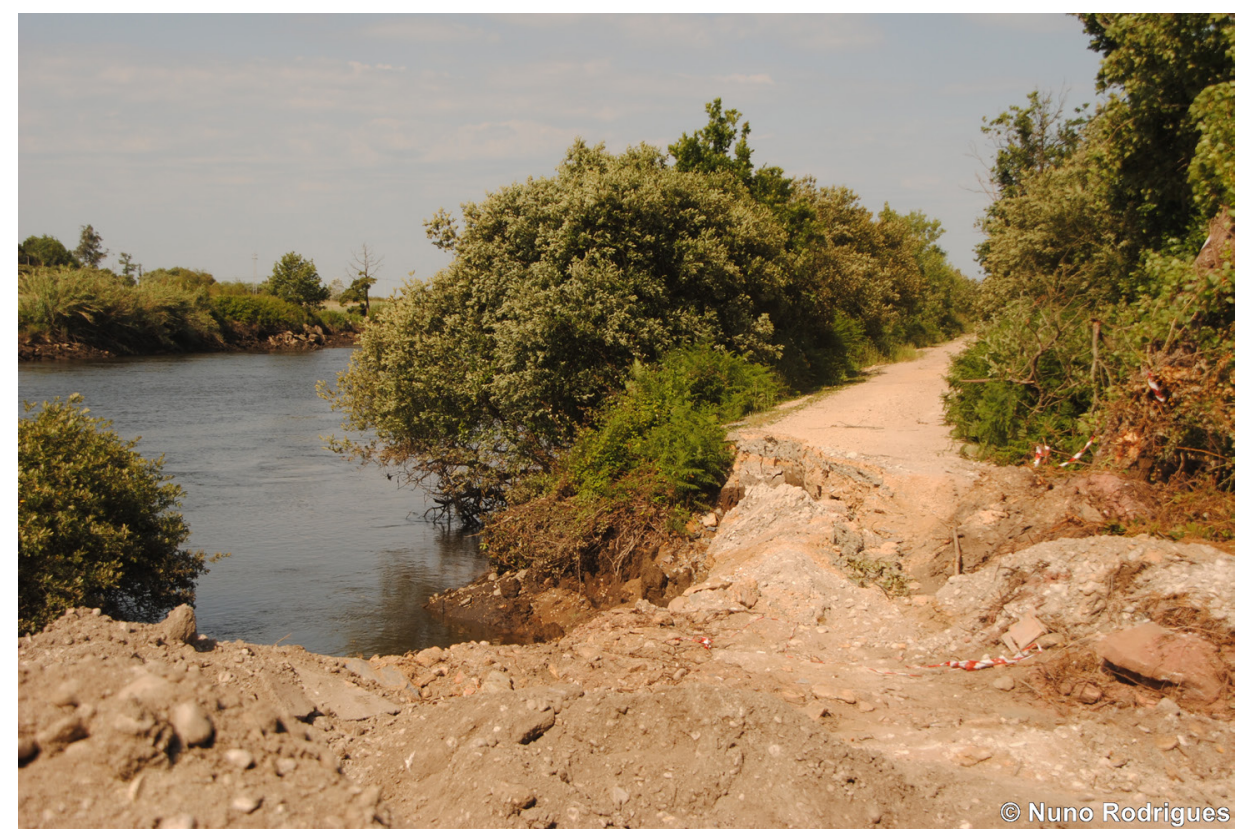

Figura 4. Colapso das margens do rio Vouga, em Cacia, em maio de 2014.

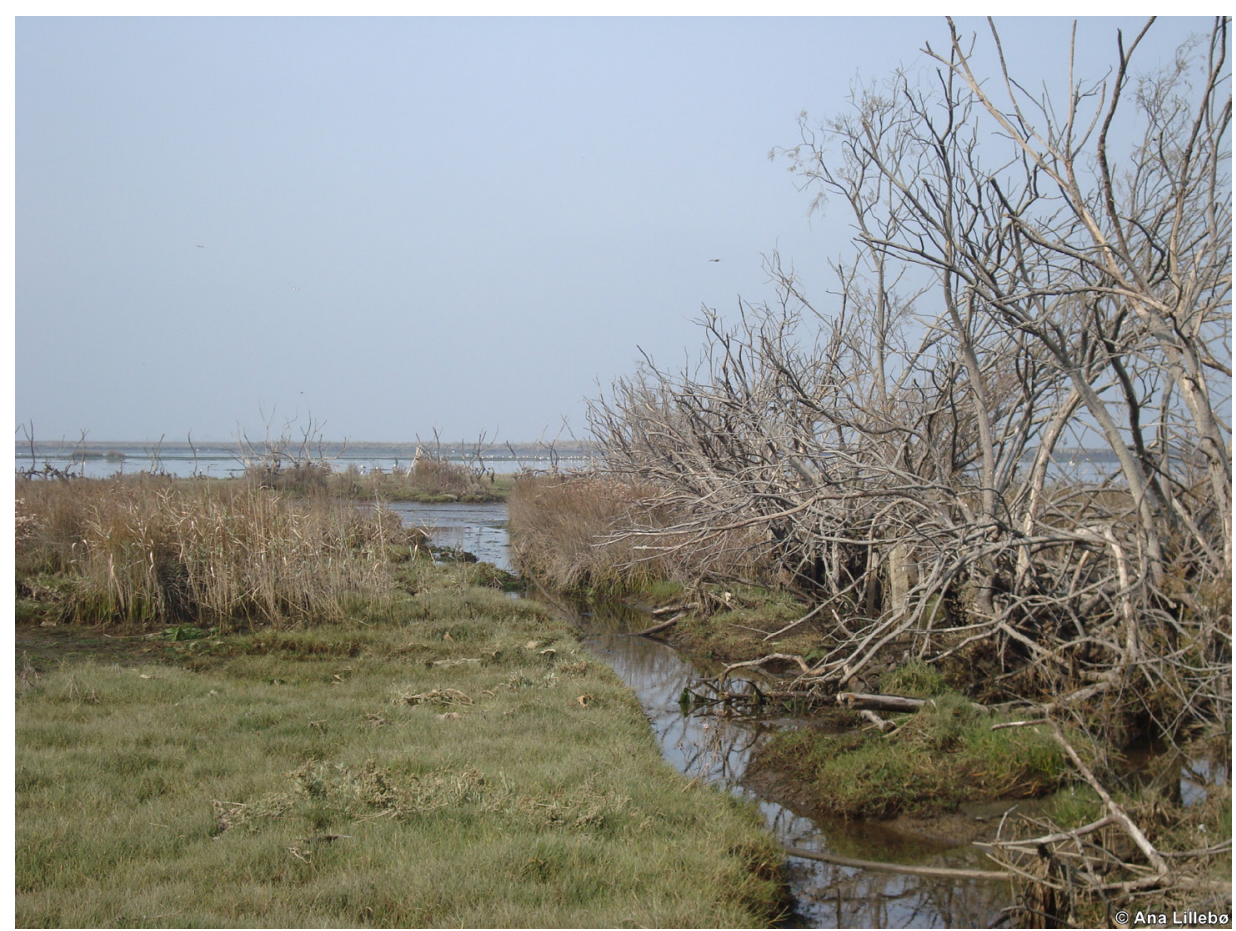

Figura 5. Exemplo do processo de degradação das sebes na paisagem do 'Bocage' entre o rio Velho e o rio Novo do Príncipe, por efeito da salinização do solo, em outubro de 2011. 


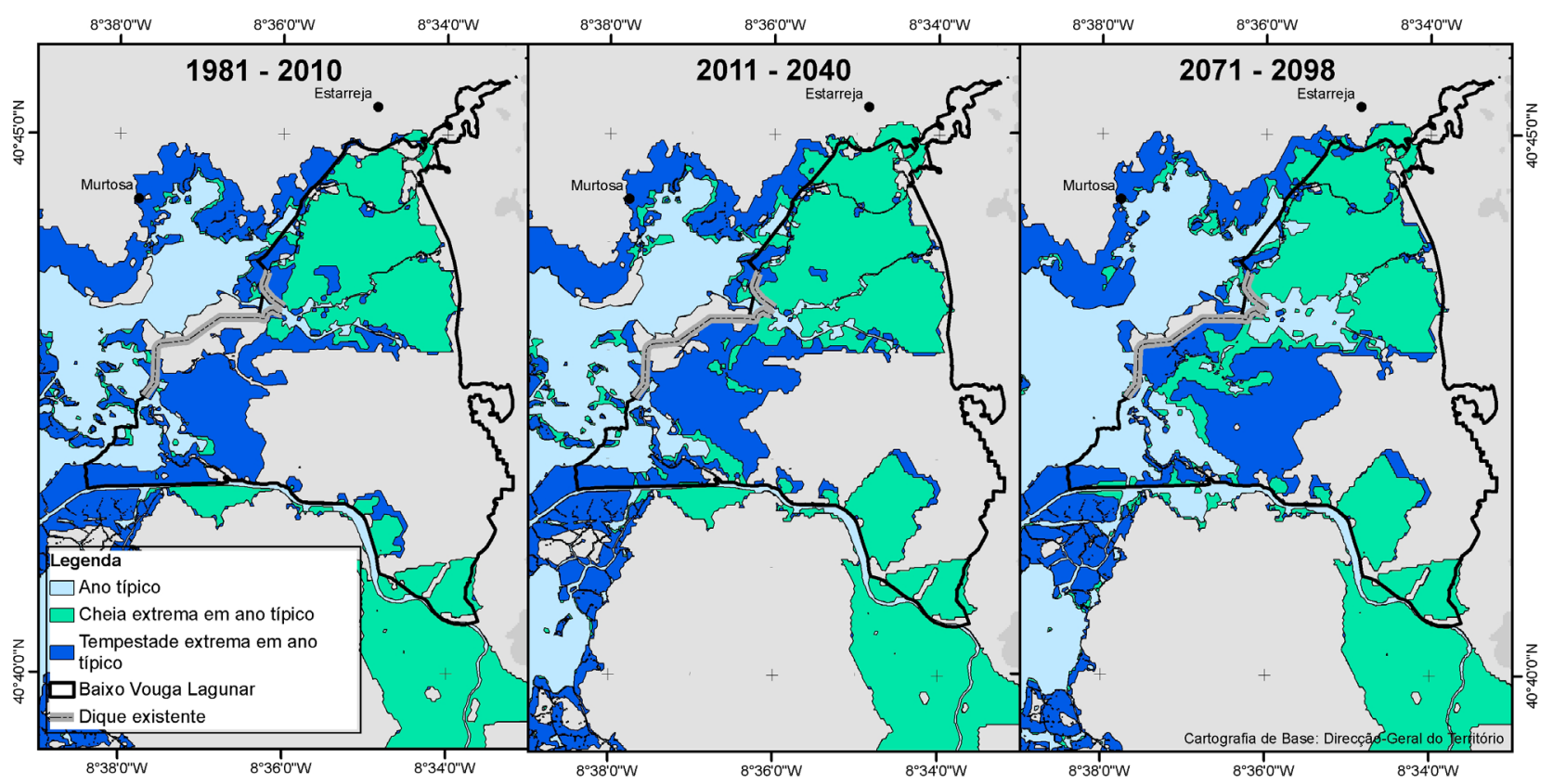

Figura 6. Cenários de inundação para o BVL (fonte: adaptado de LAGOONS, 2014).

e para os cenários de cheia associada a baixos períodos de retorno (de cheia extrema e de tempestade marítima extrema) (LAGOONS, 2014)4. É notória a vulnerabilidade do BVL face a inundações, nomeadamente em situações extremas, e o papel que o dique existente representa na contenção da água (LAGOONS, 2014). A extensão da área inundada aumenta ao longo dos anos, em todos os cenários.

Em termos do ciclo hidrológico, Stefanova et al. (2014) estimam que, para a bacia hidrográfica do Vouga, em média a precipitação anual diminuirá $8 \%$ no período de 2041-2070 e 16\% no período de 2071-2098 (em relação ao período 1971-2010). Durante os meses de verão estima-se que a descarga total para a

4 - O cenário de ano típico considera a maior maré viva, em cada período, em condições meteorológicas típicas. O cenário de cheia extrema ocorre no fim de um verão seco, considerando o caudal de cheia centenária (obtido através do ajuste de uma distribuição, em cada período de 30 anos, dos caudais dos 6 rios - ver LAGOONS (2014)), e a maior maré equacional em cada período de 30 anos. O cenário de tempestade marítima extrema ocorre no inverno, considerando, simultaneamente, o caudal de cheia centenária, a elevação máxima do nível do mar, num período de retorno de 100 anos, a maior maré equacional e a maior amplitude de maré, de cada período de 30 anos.
Ria de Aveiro diminua 10 a $15 \%$ no período de 2071-2098, o que afetará o abastecimento de água doce em áreas como o BVL, bem como os padrões de salinidade (Stefanova et al., 2014).

\subsection{Possíveis impactes sobre os serviços de ecossistema}

Considerando os cenários dealteraçãoclimática, nomeadamente no que se refere ao aumento da temperatura do ar e da água, às mudanças nos padrões da precipitação e na possibilidade do aumento da intrusão salina superficial, o ciclo hidrológico poderá ser afetado, bem como o equilíbrio entre a água doce e a salgada e, desta forma, também os serviços de ecossistema associados. Em concreto, a ocorrência de possíveis eventos de seca durante o verão, a redução da capacidade de recarga dos aquíferos e o aumento da evapotranspiração irão afetar o ciclo hidrológico do BVL e, consequentemente, os agroecossistemas e os ecossistemas de água doce. Adicionalmente, o aumento da intrusão salina superficial irá afetar principalmente os serviços associados ao "Bocage" e aos pauis pelo aumento das áreas sob a influência de água salgada, que correspondem a zonas húmidas costeiras e aos sapais sob a influência das marés e com salinidade superior a 0,5. 


\subsection{Instrumentos de Gestão Territorial}

No planeamento de medidas de adaptação há que considerar que o BVL está inserido num território complexo, constituído por ecossistemas de transição e dulçaquícolas e, desta forma, é necessário haver articulação entre as diferentes instituições públicas, nomeadamente em termos de competências e dos níveis de gestão (Alves et al., 2011; Sousa et al., 2011; ADAPT-MED, 2015a). Na Tabela 2 indicam-se as medidas de adaptação às $A C$ e medidas de gestão dos recursos hídricos que, de modo integrado, poderão funcionar como medidas de adaptação às $A C$, que se encontram já previstas nos diferentes instrumentos de gestão de territorial (IGT) aos quais o BVL está sujeito.

Tabela 2. Medidas presentes nos instrumentos de gestão territorial nos quais o BVL se encontra abrangido

(fontes: ARH Centro, 2012; CCDR, 2011; RCM n. $\left.{ }^{\circ} 115-\mathrm{A} / 2008\right)$.

\begin{tabular}{|c|c|c|}
\hline Instrumento & Âmbito & Responsabilidade de aplicação \\
\hline $\begin{array}{l}\text { Plano de Gestão das Bacias Hidrográficas dos rios Vouga, Mondego } \\
\text { e Lis }\end{array}$ & Estratégico & APA, I.P (ARHCentro) \\
\hline \multicolumn{3}{|l|}{ Medidas } \\
\hline \multicolumn{3}{|c|}{$\begin{array}{l}\text { - Controlo de espécies invasoras em habitats selecionados nas massas de água de transição } \\
\text { - Melhoria da conetividade estuarina } \\
\text { - Cumprimento da diretiva sobre o Risco de Inundações } \\
\text { - Elaborar o Plano de Ordenamento do Estuário do Vouga } \\
\text { - Construção do dique de defesa do projeto Hidroagrícola do Baixo Vouga Lagunar } \\
\text { - Estudo da evolução e da dinâmica costeira e estuarina } \\
\text { - Definição de um plano quinquenal de dragagens para a barra e canais de navegação da ria de Aveiro e sua posterior fiscalização }\end{array}$} \\
\hline Instrumento & Âmbito & Responsabilidade de aplicação \\
\hline Plano Regional de Ordenamento do Território do Centro (PROT-C) & $\begin{array}{l}\text { Estratégico e normativo } \\
\text { (por aprovar) }\end{array}$ & $\begin{array}{l}\text { Comissão de Coordenação e Desenvolvimento } \\
\text { Regional do Centro; Câmaras Municipais }\end{array}$ \\
\hline
\end{tabular}

\section{Medidas}

- Incorporação nos IGT: A avaliação, monitorização e implementação, no quadro das alterações climáticas, a modelação topo-hidrográfica da orla costeira dos regimes fluviais associados a cheias rápidas e progressivas, para horizontes temporais abrangentes; uma visão preventiva na gestão da orla costeira tendo em conta a subida do nível médio das águas do mar e as alterações climáticas

- Implementação de programas e ações de sensibilização e preparação das populações e grupos específicos para as consequências das alterações climáticas e da subida do nível médio do mar

- Garantir a construção da barragem de Ribeiradio para regularização de caudais e utilização da água para fins múltiplos

- Promover o projeto de Valorização Agrícola do Baixo Vouga Lagunar numa ótica de manutenção do mosaico de biótopos atualmente existente fomentando a atividade agrícola através da manutenção da variedade de sistemas culturais praticados, da valorização das áreas de arrozal, vegetação palustre e "Bocage" promovendo a qualidade ecológica das águas

- Garantir a conclusão do dique de defesa dos Campos Agrícolas do Vouga

- Integração do Baixo Vouga Lagunar na Rede Nacional de Áreas Protegidas

- Equacionar a criação do Parque Natural da Ria de Aveiro no âmbito da elaboração do Plano de Ordenamento do Estuário da Ria de Aveiro

- Promover e apoiar a manutenção de arrozais em regime extensivo na ZPE da Ria de Aveiro, incluindo os arrozais de Salreu

- Avaliar devidamente os efeitos das dragagens em zonas húmidas sobre os valores naturais

- Privilegiar-se o reforço da prevenção e redução da perigosidade relacionada com: Dinâmica e erosão costeira; Cheias, inundações e galgamentos marinhos (incluindo roturas parciais ou totais de barragens)

- Promover a recuperação das "motas" tradicionais de proteção

- Avaliar devidamente os efeitos das dragagens sobre os valores naturais, nomeadamente sobre os valores existentes nas zonas mais interiores da Ria (ex: Baixo Vouga), condicionando a aprovação de dragagens em cotas que coloquem em risco, devido a alterações de hidrodinâmica nos canais, os valores naturais dessas zonas 
Tabela 2. Continuação.

\begin{tabular}{|c|c|c|}
\hline Instrumento & Âmbito & Responsabilidade de aplicação \\
\hline Plano Setorial da Rede Natura 2000 & Estratégico e normativo & $\begin{array}{l}\text { Instituto Nacional da Conservação da Natureza } \\
\text { e das Florestas, I.P. (ICNF) }\end{array}$ \\
\hline \multicolumn{3}{|l|}{ Medidas } \\
\hline \multicolumn{3}{|c|}{$\begin{array}{l}\text { - Manutenção e restauro das áreas húmidas e mosaico de habitats, na Ria de Aveiro, promovendo a coexistência de habitats de alimentação (vasas } \\
\text { e salinas), habitats de nidificação e repouso (sapais) e corredores de migração } \\
\text { - No BVL, deverão ser cumpridas as exigências das boas práticas agrícolas, a monitorização da qualidade da água e a preservação das galerias } \\
\text { ripícolas } \\
\text { - Outras medidas: Conservar sebes, bosquetes e arbustos; condicionar a construção de infraestruturas; regular o uso de açudes; manter/recuperar } \\
\text { salinas; condicionar intervenções nas margens e leito de linhas de água; condicionar drenagem; recuperar zonas húmidas. }\end{array}$} \\
\hline Instrumento & Âmbito & Responsabilidade de aplicação \\
\hline Plano de Ordenamento do Estuário do Vouga & (em preparação) & APA, I.P (ARH Centro) \\
\hline \multicolumn{3}{|l|}{ Medidas } \\
\hline \multicolumn{3}{|l|}{ (em elaboração) } \\
\hline Instrumento & Âmbito & Responsabilidade de aplicação \\
\hline Planos Diretores Municipais & $\begin{array}{l}\text { Planeamento e } \\
\text { desenvolvimento } \\
\text { territorial. }\end{array}$ & Municípios, CCDR-C \\
\hline \multicolumn{3}{|l|}{ Medidas } \\
\hline $\begin{array}{l}\text { Aveiro: (sem medidas relevantes) } \\
\text { Albergaria-a-Velha: } \\
\text { - } \quad \text { Consideração do Projeto de Desenvolvimento Agrícola do Vouga }\end{array}$ & & \\
\hline $\begin{array}{l}\text { Estarreja: } \\
\text { - } \quad \text { Projeto de Aproveitamento Hidroagrícola do Vouga } \\
\text { - } \quad \text { Proteção e defesa da Zona Lagunar e revitalização de áreas natu } \\
\text { - } \quad \text { Manutenção da estrutura do "Bocage" } \\
\text { - } \quad \text { Recuperação das margens do Rio Antuã e de outras estruturas da }\end{array}$ & ais estruturantes degradadas & \\
\hline Instrumento & Âmbito & Responsabilidade de aplicação \\
\hline Unir@RIA - Plano Intermunicipal de Ordenamento da Ria de Aveiro & $\begin{array}{l}\text { Estratégico, orientações } \\
\text { para o desenvolvimento } \\
\text { estratégico regional }\end{array}$ & $\begin{array}{l}\text { Comunidade Intermunicipal da Região de } \\
\text { Aveiro (CIRA); Municípios }\end{array}$ \\
\hline \multicolumn{3}{|l|}{ Medidas } \\
\hline \multicolumn{3}{|c|}{ - Intervenções nas infraestruturas de drenagem e de proteção contra o efeito das marés } \\
\hline Instrumento & Âmbito & Responsabilidade de aplicação \\
\hline Programa Estratégico "POLIS Litoral - Ria de Aveiro" & $\begin{array}{l}\text { Intervenções de } \\
\text { recuperação e } \\
\text { reabilitação }\end{array}$ & $\begin{array}{l}\text { Sociedade de Requalificação e Valorização da } \\
\text { Ria de Aveiro S.A. - Parque-Expo, APA I.P., ICNF } \\
\text { I.P., CIRA }\end{array}$ \\
\hline \multicolumn{3}{|l|}{ Medidas } \\
\hline \multicolumn{3}{|c|}{$\begin{array}{l}\text { - Reforço das margens pela recuperação de diques motas com vista à prevenção de riscos } \\
\text { - Estudo da evolução e da dinâmica costeira e estuarina } \\
\text { - Estudo de caracterização da qualidade ecológica da ria }\end{array}$} \\
\hline
\end{tabular}

\subsection{Perceção dos atores-chave sobre 0 impacte das alterações climáticas no Baixo Vouga Lagunar}

A seleção dos entrevistados foi direcionada para os atores-chave institucionais e pertencentes a organizações não-governamentais. Nesse sentido foram contactadas 21 entidades e realizadas 18 entrevistas. Ainda que seja um número limitado, as entrevistas abrangeram diferentes grupos de atores-chave permitindo retirar algumas ilações. Em termos gerais verificou-se que não existe uma tendência clara e diferenciada entre grupos de entrevistados no que se refere às suas perceções quanto aos problemas ambientais observados (e.g. intrusão salina superficial, inundações fluviais). A análise das entrevistas permitiu constatar 
que, entre cerca de $50 \%$ dos entrevistados, as AC não constituem a maior preocupação no BVL, e $56 \%$ dos entrevistados apontam as atividades associadas ao Porto de Aveiro, nomeadamente a dragagem dos canais de navegação, como causas prováveis para os problemas ambientais observados (e.g. intrusão salina superficial). Estas perceções vão de encontro ao anteriormente mencionado por Picado et al. (2010), referindo que outros fatores, que não as $A C$, serão responsáveis pelos problemas ambientais observados.

Em detalhe, um dos dezoito entrevistados mencionou que "as alterações climáticas são um problema global e, desta forma, devem ser resolvidas a uma escala global"; outro referiu que "os problemas atuais devem ser resolvidos antes de se considerarem as alterações climáticas" e outro ainda, que a incerteza associada ao fenómeno das AC, bem como a falta de consenso sobre as assunções e as atribuições referentes aos problemas ambientais sentidos, têm funcionado como entrave à concretização de certas medidas. Cerca de $50 \%$ dos entrevistados desvaloriza as $\mathrm{AC}$, considerando que "não existem grandes consequências das alterações climáticas no BVL" ou que o impacte associado é bem menor do que as consequências das dragagens do Porto de Aveiro. Existem contudo dúvidas, incertezas e desconhecimento sobre as $A C$ e seus impactes. Neste contexto foi ainda referido que os agricultores e os produtores de gado locais não estarão sensibilizados para as AC (39\%), havendo outras questões mais prementes

As preocupações dos entrevistados encontramse, fundamentalmente, direcionadas para o impacto causado pela intrusão salina superficial, proveniente da Ria de Aveiro (78\%); o colapso das margens (72\%); as inundações extremas fluviais, de montante (72\%); o aumento do processo de erosão (44\%); as alterações no prisma da maré (39\%); as tempestades (33\%);e o acentuado recuo da linha de costa (22\%).

Verões mais frescos e chuvosos e eventos de precipitação mais intensos e frequentes foram apontados como indícios das AC. No entanto foi referido que "sempre choveu muito nesta região, por isso pode não se dever às $A C$ ". No que respeita às possíveis consequências das $A C$ mencionaram-se as alterações na paisagem (61\%), as perdas de habitat e consequente perda de biodiversidade (50\%), a redução da produtividade agrícola e do rendimento (50\%), o abandono agrícola (44\%), e ameaças às habitações (11\%).

Foram apontadas como possíveis razões para explicarainaçãofaceaosproblemasidentificados a gestão, nem sempre adequada, da Ria de Aveiro (78\%), os conflitos entre entidades (50\%) e a falta de apoio aos agricultores para lidarem com inundações fluviais e/ou com a intrusão salina superficial (proveniente da Ria de Aveiro) (33\%). As sucessivas alterações nas estruturas institucionais e a "falta de comunicação entre entidades" foram também referidas como constituindo um entrave à adoção de medidas estruturantes e de longo prazo (39\%), visando a gestão integrada do território. As medidas de mitigação sobre os impactes atuais foram identificadas como tendo sido reativas (50\%), entre elas, as intervenções de reparação dos rombos nas margens e outras iniciativas isoladas, como a instalação de comportas de maré e a reparação de pequenos diques.

Alguns dos entrevistados referiram que "não existem ainda medidas concretas para integrar as alterações climáticas nas políticas atuais" ou para evitar os impactes da intrusão salina superficial. Na sua maioria, os entrevistados reconhecem (67\%) que a conclusão do dique de defesa de marés seria uma medida exemplar para responder aos problemas atuais e futuros, ainda que o seu dimensionamento devesse ser adaptado para lidar com alguns dos efeitos das AC (e.g. subida do nível do mar).

Outros projetos e medidas foram referidos pelos entrevistados, como oportunidades de concretização de medidas de adaptação das AC nas políticas atuais. São eles: o Programa Operacional Regional "MAIS CENTRO", no âmbito do novo Quadro de Referência Estratégico Nacional aplicado aos riscos e à valorização territorial (33\%); a continuidade do programa "Polis Litoral - Ria de Aveiro" (28\%); o projeto BioRia (17\%); a conclusão do Plano de Ordenamento da Orla Costeira (POOC) (11\%); a redução da emissão de licenças de caça (6\%); e a aprovação do PROT-C (6\%).

No que se refere ao horizonte 2050, os 
entrevistados manifestaram bastante expectativa na elaboração de estudos claros e fidedignos sobre as AC (56\%) e sobre o melhoramento das estruturas de proteção face ao avanço da água e na gestão dos recursos hídricos (50\%). As medidas propostas nas entrevistas incluem a elaboração de planos de gestão da zona costeira (89\%); a construção de novos diques tendo em conta a margem de segurança e a substituição dos materiais das comportas de maré (17\%); e o reforço das dunas (6\%). Estas medidas vão ao encontro das medidas propostas por Alves et al. (2013b).

Também o melhoramento da relação entre os diversos atores (entidades reguladoras, administração, agricultores, e indústria) foi apontado como um aspeto essencial para uma abordagem integrada sobre os problemas sentidos, tal como já tinha sido reconhecido por Fidélis, Roebeling (2014), assim como "apoiar financeiramente os agricultores ativos, quer no âmbito do investimento nas suas explorações agrícolas, quer num apoio complementar ao rendimento da atividade pelo serviço que prestam à sociedade em preservarem o ambiente no território". Por fim foi realçado que "deve haver uma visão integrada relativamente às alterações climáticas, não a nível do município mas mais abrangente" por forma a integrar as AC e as de medidas de adaptação na elaboração de políticas.

\section{CONCLUSÕES}

A subida do nível médio do mar e o aumento da frequência dos eventos climáticos extremos têm sido associados às AC. No entanto, através da análise da literatura disponível e das perceções observadas dos atores-chave apurou-se que, no caso do BVL, as alterações do prisma e da amplitude da maré estão mais associadas às obras de dragagem junto ao porto de Aveiro. Ainda assim, estima-se que o aumento do nível médio do mar na costa portuguesa no fim do século se situe entre $0,42 \mathrm{~m}$ e $0,64 \mathrm{~m}$, o que para a Ria de Aveiro poderá significar que a área inundada aumente entre $23 \%$ a $35 \%$, amplificando o potencial de intrusão salina superficial e comprometendo as atuais infraestruturas e atividades (no entanto importa referir que este modelo não têm em conta os processos biogeomorfológicos de resposta por parte dos ecossistemas da laguna). Ainda que com base num número limitado de entrevistas verificou-se que as perceções dos atores chave são concordantes com as conclusões dos estudos e de projetos que têm abrangido o BVL e que foram alvo de análise no presente estudo. Efetivamente, as $A C$ não são de momento a maior preocupação de momento: os atores-chave consideram que outros problemas, concretamente a intrusão salina superficial, os rombos nas margens, as inundações fluviais de montante, as tempestades e as alterações no prisma da maré, devem ser tratados de forma prioritária.

$\mathrm{Na}$ perspetiva dos dezoito entrevistados não existem ainda medidas concretas para integrar as AC nas políticas de gestão atuais consideram, também, que a gestão da água na Ria de Aveiro não tem sido adequada para lidar com a intrusão salina superficial e com as inundações fluviais. No entanto foram destacadas medidas existentes cuja continuidade irá contribuir para uma melhor gestão da água no BVL. São exemplos dessas medidas: o Projeto Agrícola do Baixo Vouga, o Projeto BioRia, a conclusão do POOC, a aprovação do PROT-C e a continuidade do programa "Polis Litoral - Ria de Aveiro". Foi ainda sugerido que algumas destas medidas poderão ser ajustadas no sentido de favorecer a adaptação do BVL aos possíveis efeitos das AC.

\section{AGRADECIMENTOS}

O presente estudo foi desenvolvido com o financiamento do projeto ADAPT-MED (EU/ FP7 CIRCLE2-MED), através da Fundação para a Ciência e a Tecnologia (FCT) (CIRCLEMED/0001/2013; CIRCLE-MED/0002/2013). Os autores agradecem à FCT o financiamento através das unidades de investigação envolvidas (PEst-OE/EGE/UI0315/2014; UID/ $A M B / 50017 / 2013)$, e através das bolsas de doutoramento SFRH/BD/79170/2011 (Lisa P. Sousa) e SFRH/BD/78348/2011 (Fabiana Freitas).

Os autores agradecem a todos os investigadores parceiros do projeto ADAPT-MED e aos atoreschave do BVL entrevistados, por toda a disponibilidade e interesse em participar no Projeto ADAPT-MED. 


\section{BIBLIOGRAFIA}

ADAPT-MED (2015a) - Baixo Vouga Lagunar Knowledge Database. ADAPT-MED Report D2.1b. 92 p. (disponível em http://circle-2.wix. com/adapt-med/).

Alves F.L., Silva J.V., Pereira C.A. \& Sousa L.P. (2011) - Ten Years Assessment of ICZM Principles Applied at Local Scale: Ria de Aveiro Case Study. Journal of Coastal Research. SI 64, 1311-1315.

Alves F. L., Coelho C. D., Pereira C., Silva J. V. \& Sousa, L. P. (2013a) - Histórico de eventos. In: J. M. Dias e F. L. Alves (eds.), Risco de Cheia e Estratégias de Adaptação para a Zona Costeira e Lagunar da Ria de Aveiro, pp. 7-10, CESAM, UAVR. Aveiro. ISBN 978-989-98755-0-0.

Alves F. L., Coelho C. D., Antunes I.C., Sousa L. P., Pereira C., Fernandes M.L. \& Roebeling, P.C. (2013b) - Estratégias de adaptação. In: J. M. Dias e F. L. Alves (eds.), Risco de Cheia e Estratégias de Adaptação para a Zona Costeira e Lagunar da Ria de Aveiro, pp. 36-49, CESAM, UAVR. Aveiro. ISBN 978-989-98755-0-0.

Andresen T. \& Curado M. J. (2001) - Estudo de Impacte Ambiental do Projecto de Desenvolvimento Agrícola do Vouga - Bloco do Baixo Vouga Lagunar - Relatório Final. IHERA (MADRP) \& Universidade de Aveiro, Aveiro.

ARH Centro (2012) - Plano de Gestão das Bacias Hidrográficas dos rios Vouga, Mondego e Lis. Ministério da Agricultura, Mar, Ambiente e Ordenamento do Território.

Canaveira P. \& Papudo R. (2013) - Relatório de Progresso da Estratégia Nacional de Adaptação às Alterações Climáticas. 225p., Agência Portuguesa do Ambiente, I.P., Amadora.

CCDR (2011) - Plano Regional do Ordenamento do Território do centro: proposta de PROTCcentro. Comissão de Coordenação e Desenvolvimento Regional do Centro, Coimbra.

CPU (2007) - Plano Intermunicipal de Ordenamento da Ria de Aveiro: relatório do plano, versão final. 286 p.
EEA (2015) - Climate change impacts and adaptation. European Briefings from "SOER 2015 - The European environment — state and outlook 2015". In: http://www.eea.europa.eu/ soer-2015/europe/climate-change-impactsand-adaptation (acedido em 11 maio 2016).

Fidélis T. \& Roebeling P. (2014) - Water resources and land use planning in Portugal - Exploring better synergies through Ria de Aveiro. Land Use Policy, Vol. 39, 84-95.

IPCC (2013) - Climate Change 2007: The Physical Science Basis. Contribution of Working Group I to the Fourth Assessment Report of the Intergovernmental Panel on Climate Change. 996 p., Cambridge University Press, Cambridge, United Kingdom and New York, USA.

IPCC (2014a) - Climate Change 2014: Impacts, Adaptation, and Vulnerability. Global and Sectoral Aspects. Contributions of Working Group II to the Fifth Assessment Report of the Intergovernmental Panel on Climate Change. 1132p., Cambridge University Press, Cambridge, United Kingdom and New York, USA.

IPCC (2014b) - SRES emissions scenarios. In: http://sedac.ciesin.columbia.edu/ddc/sres/ (acedido em 14 maio 2014).

LAGOONS (2013) - Results of the problem based science analysis: The Ria de Aveiro Lagoon. LAGOONS Report D3.2.1. 50 p. (disponível em http://lagoons.web.ua.pt)

LAGOONS (2014) - Results of combined climate and ecosystem processes: Report describing results of combined climate and ecosystem processes. LAGOONS Report D6.3. 139 p. (disponível em http://lagoons.web.ua.pt).

Lopes C. L., Azevedo A. \& Dias J. M. (2013) - Flooding assessment under sea level rise scenarios: Ria de Aveiro case study. Journal of Coastal Research, S165, 766-771.

Lopes C. L., Silva P. A., Dias J. M., Rocha A., Picado A., Plecha S. \& Fortunato A. B. (2011) - Local sea level change scenarios for the end of the 21st century and potential physical impacts in the lower Ria de Aveiro (Portugal). Continental Shelf Research, 31, 14, 1515-1526. 
Parque Expo (2010) - Ria de Aveiro POLIS LITORAL: Requalificação e Valorização da Orla Costeira, plano estratégico. 215 p.

Picado A., Dias J. M. \& Fortunato A. B (2010) Tidal Changes in estuarine systems induced by local geomorphologic modifications. Continental Shelf Research, 30, 17, 1854-1864.

Santos F. \& Miranda P. (2006) - Alterações Climáticas em Portugal: Cenários, Impactos e Medidas de Adaptação. Projecto SIAM II. Gradiva, Lisboa.

Santos, F.D., Lopes A.M., Moniz G., Ramos L. \& Taborda R. (2014) - Gestão da Zona Costeira O Desafio da Mudança. Relatório do Grupo de Trabalho do Litoral. 260 p.

Schmidt L., Santos F. D., Prista P., Saraiva T. \& Gomes C. (2012) - Alterações climáticas, sociais e políticas em Portugal: processos de governança num litoral em risco. Ambiente \& sociedade, 15(1), 23-40. ISSN: 1809-4422.
Silva J., Duck R. \& Catarino J. (2004) - Seagrass and sediment response to changing physical forcing in a coastal lagoon. Hydrology and Earth System Sciences, 8, 151-159.

Sousa L.P., Alves F.L. \& Silva J.V. (2011) - Competing Uses on Marine Space: Methodological Aspects to Consider in Estuary Management Plans. Journal of Coastal Research. SI64, 1584-1588.

Sousa L.P., Lillebø A.I., Soares J.A. \& Alves F.L. (2015) - The management story of Ria de Aveiro. In: Lillebø A.I., Stålnacke P. \& Gooch G.D. (Eds.), Coastal Lagoons in Europe: Integrated Water Resource Strategies, pp. 31-38, IWA Publishers, London. ISBN: 9781780406282.

Stefanova A., Krysanova V., Hesse C. \& Lillebø, A. I. (2014) - Climate change impact assessment on water inflow to a coastal lagoon: the Ria de Aveiro watershed, Portugal. Hydrological Sciences Journal. 60:5, 929-948. 